\title{
The b-chromatic number of some power graphs
}

\author{
Brice Effantin ${ }^{1}$ and Hamamache Kheddouci ${ }^{2}$ \\ LE2I FRE-CNRS 2309, Université de Bourgogne, B.P. 47870, 21078 Dijon Cedex, France \\ ${ }^{1}$ brice.effantineu-bourgogne.fr \\ ${ }^{2}$ kheddoucdu-bourgogne. fr
}

received Jul 19, 2002, revised Oct 29, 2002, accepted Apr 22, 2003.

Let $G$ be a graph on vertices $v_{1}, v_{2}, \ldots, v_{n}$. The b-chromatic number of $G$ is defined as the maximum number $k$ of colors that can be used to color the vertices of $G$, such that we obtain a proper coloring and each color $i$, with $1 \leq i \leq k$, has at least one representant $x_{i}$ adjacent to a vertex of every color $j, 1 \leq j \neq i \leq k$. In this paper, we give the exact value for the $b$-chromatic number of power graphs of a path and we determine bounds for the b-chromatic number of power graphs of a cycle.

Keywords: b-chromatic number, coloring, cycle, path, power graphs

\section{Introduction}

We consider graphs without loops or multiple edges. Let $G$ be a graph with a vertex set $V$ and an edge set $E$. We denote by $d(x)$ the degree of the vertex $x$ in $G$, and by $\operatorname{dist}_{G}(x, y)$ the distance between two vertices $x$ and $y$ in $G$. The $p$-th power graph $G^{p}$ is a graph obtained from $G$ by adding an edge between every pair of vertices at distance $p$ or less, with $p \geq 1$. It is easy to see that $G^{1}=G$. In the literature, power graphs of several classes have been investigated [2, [3, 8]. In this note we study a vertex coloring of power graphs. The power graph of a path and the power graph of a cycle can be also considered as respectively subclasses of distance graphs and circulant graphs. The distance graph $G(D)$ with distance set $D=\left\{d_{1}, d_{2}, \ldots\right\}$ has the set $Z$ of integers as vertex set, with two vertices $i, j \in Z$ adjacent if and only if $|i-j| \in D$. The circulant graph can be defined as follows. Let $n$ be a natural number and let $S=\left\{k_{1}, k_{2}, \ldots, k_{r}\right\}$ with $k_{1}<k_{2}<\ldots<k_{r} \leq n / 2$. Then the vertex set of the circulant graph $G(n, S)$ is $\{0,1, \ldots, n-1\}$ and the set of neighbors of the vertex $i$ is $\left\{\left(i \pm k_{j}\right) \bmod n \mid j=1,2 \ldots, r\right\}$.

The study of distance graphs was initiated by Eggleton and al. [4]. Recently, the problem of coloring of this class of graphs has attracted considerable attention, see e.g. [12, 13]. Circulant graphs have been extensively studied and have a vast number of applications to multicomputer networks and distributed computation (see [1, [10]). The special cases we consider are the distance graph $G(D)$ with finite distance set $D=\{1,2, \ldots, p\}$ which is isomorphic to the $p$-th power graph of a path and the circulant graph $G(n, S)$ with $S=\{1,2, \ldots, p\}$ which is isomorphic to the $p$-th power graph of a cycle. 
A $k$-coloring of $G$ is defined as a function $c$ on $V(G)=\left\{v_{1}, v_{2}, \ldots, v_{n}\right\}$ into a set of colors $C=$ $\{1,2, \ldots, k\}$ such that for each vertex $v_{i}$, with $1 \leq i \leq n$, we have $c_{v_{i}} \in C$. A proper $k$-coloring is a $k$ coloring satisfying the condition $c_{x} \neq c_{y}$ for each pair of adjacent vertices $x, y \in V(G)$. A dominating proper $k$-coloring is a proper $k$-coloring satisfying the following property $P$ : for each $i, 1 \leq i \leq k$, there exists a vertex $x_{i}$ of color $i$ such that, for each $j$, with $1 \leq j \neq i \leq k$, there exists a vertex $y_{j}$ of color $j$ adjacent to $x_{i}$. A set of vertices satisfying the property $P$ is called a dominating system. Each vertex of a dominating system is called a dominating vertex. The $b$-chromatic number $\varphi(G)$ of a graph $G$ is defined as the maximum $k$ such that $G$ admits a dominating proper $k$-coloring.

The b-chromatic number was introduced in [ 7$]$. The motivation, similarly as for the previously studied achromatic number (cf. e.g. [5, 6]), comes from algorithmic graph theory. The achromatic number $\psi(G)$ of a graph $G$ is the largest number of colors which can be assigned to the vertices of $G$ such that the coloring is proper and every pair of distinct colors appears on an edge. A proper coloring of a graph $G$ using $k>\chi(G)$ colors could be improved if the vertices of two color classes could be recolored by a single color so as to obtain a proper coloring. The largest number of colors for which such a recoloring strategy is not possible is given by the achromatic number. A more versatile form of recoloring strategy would be to allow the vertices of a single color class to be redistributed among the colors of the remaining classes, so as to obtain a proper coloring. The largest number of colors for which such a recoloring strategy is not possible is given by $\varphi(G)$ (these recolorings are discussed in [ [] and [I]]). Thus $\varphi(G) \leq \psi(G)$ (also given in [7]). From this point of view, both complexity results and tight bounds for the b-chromatic number are interesting. The following bounds of $b$-chromatic number are already presented in [ [7].

Proposition 1 Assume that the vertices $x_{1}, x_{2}, \ldots, x_{n}$ of $G$ are ordered such that $d\left(x_{1}\right) \geq d\left(x_{2}\right) \geq \ldots \geq$ $d\left(x_{n}\right)$. Then $\varphi(G) \leq m(G) \leq \Delta(G)+1$, where $m(G)=\max \left\{1 \leq i \leq n: d\left(x_{i}\right) \geq i-1\right\}$ and $\Delta(G)$ is the maximum degree of $G$.

R. W. Irving and D. F. Manlove [7] proved that finding the b-chromatic number of any graph is a NP-hard problem, and they gave a polynomial-time algorithm for finding the b-chromatic number of trees. Kouider and Mahéo [9] gave some lower and upper bounds for the b-chromatic number of the cartesian product of two graphs. They gave, in particular, a lower bound for the b-chromatic number of the cartesian product of two graphs where each one has a stable dominating system. More recently in [II], the authors characterized bipartite graphs for which the lower bound on the b-chromatic number is attained and proved the NP-completeness of the problem to decide whether there is a dominating proper $k$-coloring even for connected bipartite graphs and $k=\Delta(G)+1$. They also determine the asymptotic behavior for the b-chromatic number of random graphs.

In this paper, we present several exact values and determine bounds for the b-chromatic number of power graphs of paths and cycles.

Let $\operatorname{Diam}(G)$ be the diameter of a graph $G$, defined as the maximum distance between any pair of vertices of $G$. Let us begin with the following observation.

Fact 2 For any graph $G$ of order $n$, if $\operatorname{Diam}(G) \leq p$, then $\varphi\left(G^{p}\right)=n$, with $p \geq 2$.

Proof. If $\operatorname{Diam}(G) \leq p$, it is trivial to see that $G^{p}$ is a complete graph. So $\varphi\left(G^{p}\right)=n$.

Let $G$ be a path or a cycle on vertices $x_{1}, x_{2}, \ldots, x_{n}$. We fix an orientation of $G$ (left to right if $G$ is a path and clockwise if $G$ is a cycle). For each $1 \leq i \leq n$, we denote by $x_{i}^{+}$(resp. $x_{i}^{-}$) the successor (resp. 
predecessor) of $x_{i}$ in $G$ (if any). For $1 \leq i \neq j \leq n$, we define $\left[x_{i}, x_{j}\right]_{G},\left[x_{i}, x_{j}\right)_{G}$ and $\left(x_{i}, x_{j}\right)_{G}$ as the set of consecutive vertices on $G$ from respectively $x_{i}$ to $x_{j}, x_{i}$ to $x_{j}^{-}$and $x_{i}^{+}$to $x_{j}^{-}$, following the fixed orientation of $G$. If there is no ambiguity, we denote $\left[x_{i}, x_{j}\right]_{G},\left[x_{i}, x_{j}\right)_{G}$ and $\left(x_{i}, x_{j}\right)_{G}$ by respectively $\left[x_{i}, x_{j}\right],\left[x_{i}, x_{j}\right)$ and $\left(x_{i}, x_{j}\right)$.

In all figures, the graph $G$ is represented with solid edges. Edges added in a $p$-th power graph $G^{p}$ are represented with dashed edges. In some figures, vertices are surrounded and represent a dominating system of the coloring. In any coloring of a graph $G$, we will say that a vertex $x$ of $G$ is adjacent to a color $i$ if there exists a neighbor of $x$ which is colored by $i$.

\section{Power Graph of a Path}

In this section, we determine the b-chromatic number of a $p$-th power graph of a path, with $p \geq 1$. First we give a lemma used in the proof of Theorem 4 . Then the b-chromatic number of a $p$-th power graph of a path is computed.

Lemma 3 For any $p \geq 1$, and for any $n \geq p+1$, let $P_{n}$ be the path on vertices $x_{1}, x_{2}, \ldots, x_{n}$. For each integer $k$, with $p+1 \leq k \leq \min (2 p+1, n)$, there exists a proper $k$-coloring on $P_{n}^{p}$. Moreover each vertex $x$, such that $x \in\left\{x_{k-p}, x_{k-p+1}, \ldots, x_{n-k+p+1}\right\}$, is adjacent to each color $j$, with $1 \leq j \neq c_{x} \leq k$.

Proof. As $k \geq p+1$, it is easy to see that if we put the set of colors $\{1,2, \ldots, k\}$ cyclically on $V\left(P_{n}\right)$, then two adjacent vertices will not have the same color. The coloring is thus a proper $k$-coloring.

Let $S=\left\{x_{k-p}, x_{k-p+1}, \ldots, x_{n-k+p+1}\right\}$. First we show that each vertex of $S$ is adjacent to at least $k-1$ vertices. Observe that the vertex $x_{k-p}$ is adjacent to $(k-p-1)+p=k-1$ vertices. And the vertex $x_{n-k+p+1}$ is adjacent to $p+n-(n-k+p+1)=k-1$ vertices. Since each vertex $x_{i}$, with $k-p+1 \leq i \leq n-k+p$, has a degree $d\left(x_{i}\right) \geq d\left(x_{k-p}\right)$, then each vertex of $S$ is adjacent to at least $k-1$ other vertices.

Next, we can see by the construction that all the colors $\{1,2, \ldots, k\} \backslash\left\{c_{x_{i}}\right\}$ appear between the first and the last neighbor of $x_{i}$. Therefore each vertex $x_{i}$ of $S$ is adjacent to each color $j$, with $1 \leq j \neq c_{x_{i}} \leq k$ and $k-p \leq i \leq n-k+p+1$.

The b-chromatic number of a $p$-th power graph of a path is given by:

Theorem 4 Let $P_{n}$ be a path on vertices $x_{1}, x_{2}, \ldots, x_{n}$. The $b$-chromatic number of $P_{n}^{p}$, with $p \geq 1$, is given by:

$$
\varphi\left(P_{n}^{p}\right)=\left\{\begin{array}{l}
n \text { if } n \leq p+1 \\
p+1+\left\lfloor\frac{n-p-1}{3}\right\rfloor \text { if } p+2 \leq n \leq 4 p+1 \\
2 p+1 \text { if } n \geq 4 p+2
\end{array}\right.
$$

Proof.

1. If $n \leq p+1$, then $\operatorname{Diam}\left(P_{n}\right) \leq p$. So, by Fact $2, \varphi\left(P_{n}^{p}\right)=n$.

2. We prove first that $\varphi\left(P_{n}^{p}\right) \geq p+1+\left\lfloor\frac{n-p-1}{3}\right\rfloor$ for $p+2 \leq n \leq 4 p+1$. Let $k=p+1+\left\lfloor\frac{n-p-1}{3}\right\rfloor$. By Lemma 3 , we give a proper $k$-coloring of $P_{n}^{p}$. For example, Figure 1 shows a dominating proper 5-coloring of $P_{8}^{3}$. 


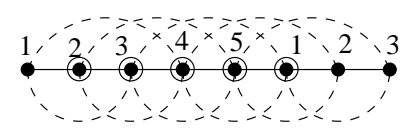

Fig. 1: Coloring of $P_{8}^{3}$

Let $S^{\prime}$ be the set of vertices $\left\{x_{k-p}, x_{k-p+1}, \ldots, x_{2 k-p-1}\right\}$. Since $2 k-p-1 \leq n-k+p+1$, then $S^{\prime} \subseteq\left\{x_{k-p}, x_{k-p+1}, \ldots, x_{n-k+p+1}\right\}$. By Lemma $3, S^{\prime}$ is a dominating system. As the coloring is proper and has a dominating system, we obtain a dominating proper $k$-coloring. So, $\varphi\left(P_{n}^{p}\right) \geq$ $p+1+\left\lfloor\frac{n-p-1}{3}\right\rfloor$.

Next we prove that $\varphi\left(P_{n}^{p}\right) \leq p+1+\left\lfloor\frac{n-p-1}{3}\right\rfloor$ for $p+2 \leq n \leq 4 p+1$. The proof is by contradiction. Suppose that there exists a dominating proper $k^{\prime}$-coloring such that

$$
k^{\prime}>p+1+\left\lfloor\frac{n-p-1}{3}\right\rfloor \text {. }
$$

Let $W=\left\{w_{1}, w_{2}, \ldots, w_{k^{\prime}}\right\}$ be a dominating system of the coloring on $P_{n}^{p}$ (following the orientation of $P_{n}$, we meet $\left.w_{1}, w_{2}, \ldots, w_{k^{\prime}}\right)$. The vertices $w_{1}$ and $w_{k^{\prime}}$ are adjacent to, at most, $p$ different colors in $\left[w_{1}, w_{k^{\prime}}\right]$. As $w_{1}$ (respectively $w_{k^{\prime}}$ ) is a dominating vertex, it must be adjacent to at least $k^{\prime}-1$ different colors. Then, there are at least $k^{\prime}-p-1$ vertices on $\left[x_{1}, w_{1}\right)$ (respectively $\left(w_{k^{\prime}}, x_{n}\right]$ ). Therefore, $n-k^{\prime} \geq n-\left|\left[w_{1}, w_{k^{\prime}}\right]\right| \geq 2\left(k^{\prime}-p-1\right)$.

On the other hand by hypothesis $k^{\prime} \geq p+2+\left\lfloor\frac{n-p-1}{3}\right\rfloor$, so that $n-k^{\prime} \leq n-p-2-\left\lfloor\frac{n-p-1}{3}\right\rfloor$.

These two results give the following inequality,

$$
\begin{gathered}
2\left(k^{\prime}-p-1\right) \leq n-k^{\prime} \leq n-p-2-\left\lfloor\frac{n-p-1}{3}\right\rfloor, \\
k^{\prime} \leq \frac{1}{2}\left(n+p-\left\lfloor\frac{n-p-1}{3}\right\rfloor\right) .
\end{gathered}
$$

By (II) and (2), we obtain,

$$
\begin{gathered}
\frac{1}{2}\left(n+p-\left\lfloor\frac{n-p-1}{3}\right\rfloor\right) \geq k^{\prime} \geq p+2+\left\lfloor\frac{n-p-1}{3}\right\rfloor, \\
n-p-4 \geq 3\left\lfloor\frac{n-p-1}{3}\right\rfloor,
\end{gathered}
$$

which is a contradiction. Hence such a coloring does not exist. Therefore, $\varphi\left(P_{n}^{p}\right) \leq p+1+$ $\left\lfloor\frac{n-p-1}{3}\right\rfloor$.

We deduce from these two parts that $\varphi\left(P_{n}^{p}\right)=p+1+\left\lfloor\frac{n-p-1}{3}\right\rfloor$. 
3. $\Delta\left(P_{n}^{p}\right)=2 p$, so by Proposition 1, $\varphi\left(P_{n}^{p}\right) \leq 2 p+1$. Lemma 3 gives a proper $(2 p+1)$-coloring and shows that each vertex $x$ of the set $\left\{x_{p+1}, x_{p+2}, \ldots, x_{3 p+1}\right\}$ is adjacent to each color $j$ with $1 \leq j \neq c_{x} \leq k$. So this set is a dominating system and $\varphi\left(P_{n}^{p}\right) \geq 2 p+1$. Therefore $\varphi\left(P_{n}^{p}\right)=2 p+1$. For example, Figure 2 gives a dominating proper 7-coloring of $P_{15}^{3}$.

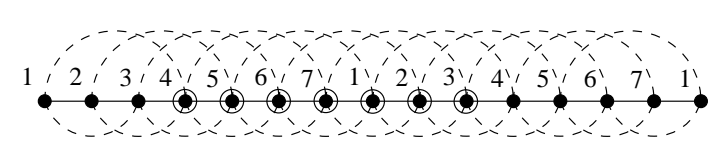

Fig. 2: Coloring of $P_{15}^{3}$

\section{Power Graph of a Cycle}

In this section, we study the b-chromatic number of a $p$-th power graph of a cycle, with $p \geq 1$. First we give two lemmas used in the proof of Theorem 7 . Then we bound the b-chromatic number of a $p$-th power graph of a cycle.

Lemma 5 Let $C_{n}^{p}$ be a $p$-th power graph of a cycle $C_{n}$, with $p \geq 2$. For any $2 p+3 \leq n \leq 4 p$, let $k \geq$ $\min \left(n-p-1, p+1+\left\lfloor\frac{n-p-1}{3}\right\rfloor\right)$. Then $n \leq 2 k$.

Proof. The proof is by contradiction. Suppose $n \geq 2 k+1$. We consider two cases. Firstly, $k \geq n-p-1$. So,

$$
\begin{aligned}
n \geq 2 k+1 & \geq 2(n-p-1)+1, \\
n & \leq 2 p+1,
\end{aligned}
$$

which is a contradiction. Secondly, $k \geq p+1+\left\lfloor\frac{n-p-1}{3}\right\rfloor$. So,

$$
\begin{gathered}
n \geq 2 k+1 \geq 2\left(p+1+\left\lfloor\frac{n-p-1}{3}\right\rfloor\right)+1, \\
n-2 p-3 \geq 2\left\lfloor\frac{n-p-1}{3}\right\rfloor,
\end{gathered}
$$

which is a contradiction too.

Lemma 6 For any $p \geq 2$, and for any $2 p+3 \leq n \leq 4 p$, let $C_{n}$ be the cycle on vertices $x_{1}, x_{2}, \ldots, x_{n}$. Let $k=\min \left(n-p-1, p+1+\left\lfloor\frac{n-p-1}{3}\right\rfloor\right)$. So there exists a proper $k$-coloring on $C_{n}^{p}$. Moreover each vertex $x$, such that $x \in\left\{x_{k-p}, x_{k-p+1}, \ldots, x_{2 k-p-1}\right\}$, is adjacent to each color $j$, with $1 \leq j \neq c_{x} \leq k$.

Proof. We put the set of colors $\{1,2, \ldots, k\}$ cyclically on $V\left(C_{n}\right)$. As $k \leq p+1+\left\lfloor\frac{n-p-1}{3}\right\rfloor$ and $n \leq 4 p$, then $k \leq 2 p+1$. Moreover, by Lemma 5 we deduce that $2 k \geq n \geq 2 p+3 \geq k+2$. So, the full set of colors $\{1,2, \ldots, k\}$ appears consecutively at least once, and at most twice, in the cyclic coloring of $C_{n}^{p}$. As $2 k \geq n \geq 2 p+3$, we have $k \geq p+1$. Furthermore, by definition of $k$ we have $n-k \geq p+1$. Thus, as $k \geq p+1$ and $n-k \geq p+1$, the coloring is proper. 
Let $P_{n}$ be the subpath of $C_{n}$ induced by $x_{1}, x_{2}, \ldots, x_{n}$. Let $S=\left\{x_{k-p}, x_{k-p+1}, \ldots, x_{k+(k-p-1)}\right\}$. As $p+1 \leq$ $k \leq 2 p+1$ and $2 k-p-1 \leq n-k+p+1$, then by Lemma 3 each vertex $x_{i}$ of $S$, with $k-p \leq i \leq 2 k-p-1$, is adjacent to each color $q$, with $1 \leq q \neq c_{x_{i}} \leq k$, on $P_{n}^{p}$. Therefore each vertex $x_{i}$ of $S$ is adjacent to each color $q$, with $1 \leq q \neq c_{x_{i}} \leq k$, on $C_{n}^{p}$.

Theorem 7 Let $C_{n}$ be a cycle on vertices $x_{1}, x_{2}, \ldots, x_{n}$. The b-chromatic number of $C_{n}^{p}$, with $p \geq 1$, is

$$
\varphi\left(C_{n}^{p}\right)=\left\{\begin{array}{l}
n \text { if } n \leq 2 p+1 \\
p+1 \text { if } n=2 p+2 \\
(\geq) \min \left(n-p-1, p+1+\left\lfloor\frac{n-p-1}{3}\right\rfloor\right) \text { if } 2 p+3 \leq n \leq 3 p \\
p+1+\left\lfloor\frac{n-p-1}{3}\right\rfloor \text { if } 3 p+1 \leq n \leq 4 p \\
2 p+1 \text { if } n \geq 4 p+1
\end{array}\right.
$$

\section{Proof.}

1. If $n \leq 2 p+1$, then $\operatorname{Diam}\left(C_{n}\right) \leq p$. So, by Fact ฤ, $\varphi\left(C_{n}^{p}\right)=n$.

2. To color the graph, we put the set of colors $\{1,2, \ldots, p+1\}$ cyclically twice. One can easily see that this coloring is a proper $(p+1)$-coloring. Let $S$ be the set of vertices $\left\{x_{1}, x_{2}, \ldots, x_{p+1}\right\}$. Each vertex $x_{i}$, with $1 \leq i \leq p+1$, is adjacent to $n-2$ vertices. Since $n-2 \geq p+1$, then each vertex $x_{i}(1 \leq i \leq p+1)$ of $S$ is adjacent to all colors other than $c_{x_{i}}$. So the set $S$ is a dominating system. We now show that, in any dominating proper coloring, vertices $x_{i}$ and $x_{i+p+1}$ must have the same color. For the subgraph induced by vertices $x_{1}, x_{2}, \ldots, x_{p+1}$, we have a clique and we can assume without loss of generality that these vertices are colored by $1,2, \ldots, p+1$ respectively. If there exists a dominating vertex of color $j$, for some $j>p+1$, then this vertex is $x_{p+1+i}$ for some $i$ $(1 \leq i \leq p+1)$. Vertex $x_{p+1+i}$ is not adjacent to $x_{i}$, but every other vertex is adjacent to $x_{i}$, so that $x_{p+1+i}$ cannot be a dominating vertex, a contradiction. Therefore $\varphi\left(C_{n}^{p}\right)=p+1$ for $n=2 p+2$.

3. Let $k=\min \left(n-p-1, p+1+\left\lfloor\frac{n-p-1}{3}\right\rfloor\right)$.

By Lemma 6 there exists a dominating proper $k$-coloring for $2 p+3 \leq n \leq 3 p$. Therefore $\varphi\left(C_{n}^{p}\right) \geq$ $\min \left(n-p-1, p+1+\left\lfloor\frac{n-p-1}{3}\right\rfloor\right)$. For example, in Figure $\beta$, we give a dominating proper 6-coloring of $C_{11}^{4}$.

4. Let $k=p+1+\left\lfloor\frac{n-p-1}{3}\right\rfloor$.

For $3 p+1 \leq n \leq 4 p$, Lemma 6 gives a dominating proper $k$-coloring. This proves that $\varphi\left(C_{n}^{p}\right) \geq$ $\min \left(n-p-1, p+1+\left\lfloor\frac{n-p-1}{3}\right\rfloor\right)$. For example, Figure 4 shows a dominating proper 6-coloring of $C_{11}^{3}$.

Next, we prove that $\varphi\left(C_{n}^{p}\right) \leq k$. Suppose there exists a dominating proper $k^{\prime}$-coloring for $C_{n}^{p}$, with $k^{\prime} \geq p+2+\left\lfloor\frac{n-p-1}{3}\right\rfloor$, for the sake of contradiction. Let $W=\left\{w_{1}, w_{2}, \ldots, w_{k^{\prime}}\right\}$ be a set of dominating vertices on $C_{n}$ (following the orientation of $C_{n}$, we meet $w_{1}, w_{2}, \ldots, w_{k^{\prime}}$ ). We distinguish two cases. 


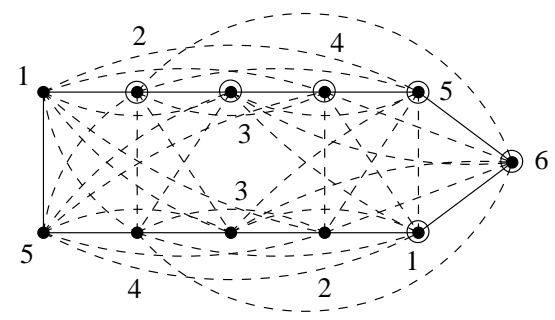

Fig. 3: Coloring of $C_{11}^{4}\left(n-p-1=6, p+1+\left\lfloor\frac{n-p-1}{3}\right\rfloor=7\right.$ and $\left.\varphi\left(C_{11}^{4}\right) \geq 6\right)$

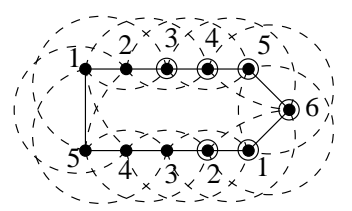

Fig. 4: Coloring of $C_{11}^{3}$

Case 1: for each $i$, with $1 \leq i \leq k^{\prime},\left|\left(w_{i}, w_{i+1}\right)\right| \leq p-1$.

As $k^{\prime} \geq p+2+\left\lfloor\frac{n-p-1}{3}\right\rfloor$, by a straightforward modification of the proof of Lemma 5 , we have $n<2 k^{\prime}$. So, there exists at least one color $c$ not repeated in $C_{n}^{p}$ (i.e. there are not two distinct vertices with the same color $c$ ). Without loss of generality, suppose that $c$ appears on the vertex $x$, with $x \in V\left(C_{n}\right)$. Therefore $x$ is a dominating vertex and each other dominating vertex is adjacent to $x$. Then, $\left|\left[w_{1}, x\right)\right| \leq p$ and $\left|\left[x, w_{k^{\prime}}\right)\right| \leq p$. As for each $i$, with $1 \leq i \leq k^{\prime}$, we have $\left|\left(w_{i}, w_{i+1}\right)\right| \leq p-1$ and since on the cycle the next dominating vertex from $w_{k^{\prime}}$ is $w_{1}$, then

$$
\left|\left(w_{k^{\prime}}, w_{1}\right)\right| \leq p-1
$$

where

$$
\left|\left(w_{k^{\prime}}, w_{1}\right)\right|=n-\left|\left[w_{1}, x\right)\right|-\left|\left[x, w_{k^{\prime}}\right)\right|-1
$$

Therefore, we have

$$
\begin{gathered}
n-\left|\left[w_{1}, x\right)\right|-\left|\left[x, w_{k^{\prime}}\right)\right|-1 \leq p-1, \\
n-2 p-1 \leq p-1, \\
n \leq 3 p
\end{gathered}
$$

which is a contradiction.

Case 2: There exists $r$, with $1 \leq r \leq k^{\prime}$ and $r$ is taken modulo $k^{\prime}$, such that $\left|\left(w_{r}, w_{r+1}\right)\right| \geq p$.

Let $X$ be the set of vertices of $\left[w_{r+1}, w_{r}\right]$ (see Figure [5). Let $X_{C}$ be the set of colors appearing in $X$. Let $\Gamma_{X}\left(x_{i}\right)$ be the set of neighbors of $x_{i}$ in $X$ and $\Gamma_{X}^{c}\left(x_{i}\right)$ the set of colors appearing in $\Gamma_{X}\left(x_{i}\right)$, with $1 \leq i \leq n$. Let $A=X_{C} \backslash\left(\Gamma_{X}^{c}\left(w_{r}\right) \cup\left\{c_{w_{r}}\right\}\right)$. Let $B=X_{C} \backslash\left(\Gamma_{X}^{c}\left(w_{r+1}\right) \cup\left\{c_{w_{r+1}}\right\}\right)$. We discuss two subcases. 


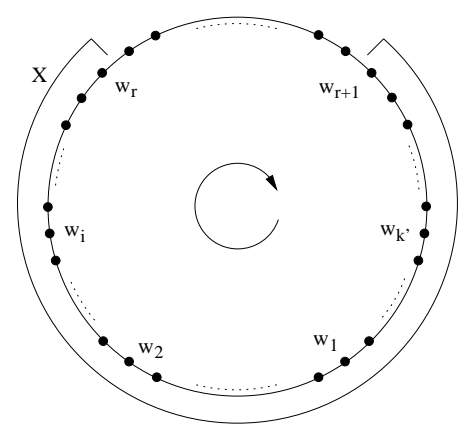

Fig. 5: A dominating system on $C_{n}^{p}$ and the set $\mathrm{X}$

Subcase 1: $|X| \leq 2 p+2$. Since all dominating vertices belong to $X$, we have $|X| \geq k^{\prime}$. Then, $\left|\left(w_{r}, w_{r+1}\right)\right| \leq n-k^{\prime}$ and $\left|X_{c}\right|=k^{\prime}$. As the vertices of $\Gamma_{X}\left(w_{r}\right)$ form a clique, then $\left|\Gamma_{X}^{c}\left(w_{r}\right)\right|=$ $\left|\Gamma_{X}\left(w_{r}\right)\right|=p$. So we have $|A|=\left|X_{C}\right|-\left|\Gamma_{X}^{c}\left(w_{r}\right)\right|-1=k^{\prime}-p-1$. In the same way, we deduce that $|B|=k^{\prime}-p-1$. As $|X| \leq 2 p+2$, we have $X \subseteq\left(\Gamma_{X}\left(w_{r}\right) \cup \Gamma_{X}\left(w_{r+1}\right) \cup\left\{w_{r}, w_{r+1}\right\}\right)$ (see Figure 6.a). So, $A \subseteq\left(\Gamma_{X}^{c}\left(w_{r+1}\right) \cup\left\{c_{w_{r+1}}\right\}\right)$ and $B \subseteq\left(\Gamma_{X}^{c}\left(w_{r}\right) \cup\left\{c_{w_{r}}\right\}\right)$. Let $q \in\left\{1,2, \ldots, k^{\prime}\right\}$. If $q \in A$ (resp. $q \in B$ ) then $q \notin\left(\Gamma_{X}^{c}\left(w_{r}\right) \cup\left\{c_{w_{r}}\right\}\right)$ (resp. $q \notin\left(\Gamma_{X}^{c}\left(w_{r+1}\right) \cup\left\{c_{w_{r+1}}\right\}\right)$ ) and so $q \notin B$ (resp. $q \notin A$ ). Therefore, $A \cap B=\emptyset$. As $w_{r}$ (resp. $\left.w_{r+1}\right)$ is a dominating vertex and $\left|\left(w_{r}, w_{r+1}\right)\right| \geq p$, the colors of $A$ (resp. $B)$ must be repeated in $\left(w_{r}, w_{r+1}\right)$. Therefore,

$$
\begin{gathered}
|A|+|B| \leq\left|\left(w_{r}, w_{r+1}\right)\right|, \\
2\left(k^{\prime}-p-1\right) \leq n-k^{\prime}, \\
3 k^{\prime} \leq n+2 p+2, \\
3\left\lfloor\frac{n-p-1}{3}\right\rfloor \leq n-p-4,
\end{gathered}
$$

which is a contradiction.

Subcase 2: $|X| \geq 2 p+3$. As in Subcase 1, we have $|A|=k^{\prime}-p-1$ and $|B|=k^{\prime}-p-1$. Let $X^{\prime}=X \backslash\left(\Gamma_{X}\left(w_{r}\right) \cup \Gamma_{X}\left(w_{r+1}\right) \cup\left\{w_{r}, w_{r+1}\right\}\right)$ (see Figure 6.b). So $\left|X^{\prime}\right| \geq|A \cap B|$. Since $w_{r}$ (resp. $\left.w_{r+1}\right)$ is a dominating vertex and $\left|\left(w_{r}, w_{r+1}\right)\right| \geq p$, the colors of $A$ (resp. $B$ ) must be repeated in $\left(w_{r}, w_{r+1}\right)$. Then,

$$
\begin{gathered}
|A|+|B|-|A \cap B| \leq\left|\left(w_{r}, w_{r+1}\right)\right| \leq n-2 p-2-\left|X^{\prime}\right|, \\
2\left(k^{\prime}-p-1\right)-|A \cap B| \leq n-2 p-2-|A \cap B|, \\
2\left(p+2+\left\lfloor\frac{n-p-1}{3}\right\rfloor\right) \leq n,
\end{gathered}
$$

which is a contradiction. Therefore there does not exist a dominating proper $k^{\prime}$-coloring, with $k^{\prime} \geq p+2+\left\lfloor\frac{n-p-1}{3}\right\rfloor$.

This completes the proof of $\varphi\left(C_{n}^{p}\right)=p+1+\left\lfloor\frac{n-p-1}{3}\right\rfloor$. 


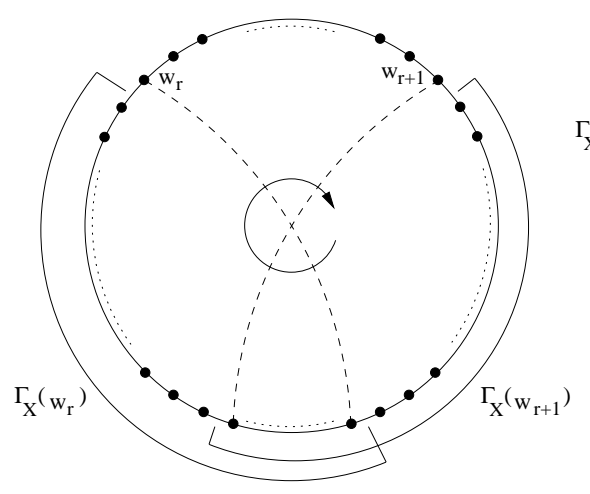

a)

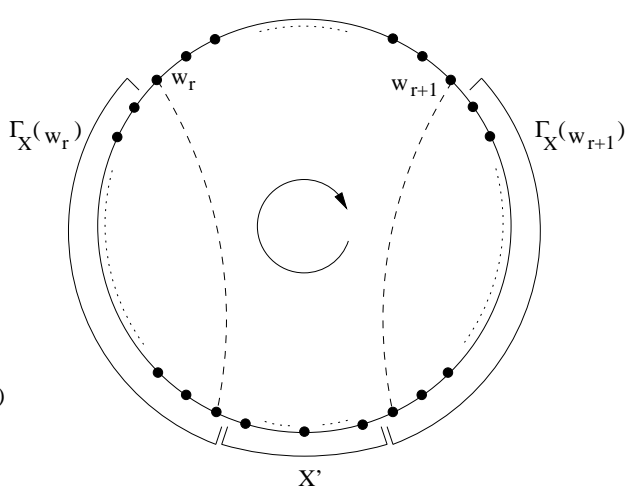

b)

Fig. 6: Neighborhoods of $w_{r}$ and $w_{r+1}$ on $X$ when a) $|X| \leq 2 p+2$ and b) $|X| \geq 2 p+3$

5. As $\Delta=2 p$, by Proposition 1, $\varphi\left(C_{n}^{p}\right) \leq 2 p+1$.

We then give a proper $(2 p+1)$-coloring. It is constructed in two steps. First, we put $(2 p+1)$ different colors on the $(2 p+1)$ first vertices $\left(c_{x_{i}}:=i\right.$ for $\left.1 \leq i \leq 2 p+1\right)$. In the second step, we have two cases. If $n=4 p+1$, we color the remaining vertices as follows: $c_{x_{i}}:=c_{x_{i-2 p-1}}$ for $2 p+2 \leq i \leq n$. If $n \geq 4 p+2$, then the remaining vertices are colored as follows: $c_{x_{i}}:=c_{x_{i-2 p-1}}$ for $2 p+2 \leq i \leq 4 p+2$, and $c_{x_{i}}:=c_{x_{i-p-1}}$ for $4 p+3 \leq i \leq n$. Then the distance between two vertices colored by the same color $c$ is at least $p+1$. So the coloring is proper. By an analogue proof of Lemma 3, one can prove that each vertex $x_{i}$, with $p+1 \leq i \leq 3 p+1$, is a dominating vertex. So this coloring is a dominating proper $(2 p+1)$-coloring. This construction shows that $\varphi\left(C_{n}^{p}\right) \geq 2 p+1$. Therefore we have proved that $\varphi\left(C_{n}^{p}\right)=2 p+1$. For example, Figure 7 gives a dominating proper 7-coloring $C_{16}^{3}$.

\section{Open Problem}

In section 3, we have obtained the exact values of $\varphi\left(C_{n}^{p}\right)$, except in case $2 p+3 \leq n \leq 3 p$ where we give a lower bound. We believe that $\min \left(n-p-1, p+1+\left\lfloor\frac{n-p-1}{3}\right\rfloor\right)$ is the exact value of $\varphi\left(C_{n}^{p}\right)$ for $2 p+3 \leq n \leq 3 p$.

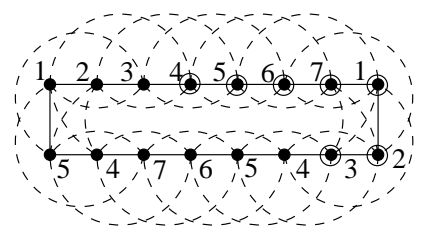

Fig. 7: Coloring of $C_{16}^{3}$ 


\section{Acknowledgments}

The authors thank the referees for useful suggestions that led to this improved version.

\section{References}

[1] J.-C. Bermond, F. Cormellas, D. F. Hsu, Distributed loop computer networks: a survey, Journal of Parallel and Distributed Computing 24 (1995) 2-10.

[2] A. BrandstäDt, V. D. Chepoi, F. F. DRagan, Perfect elimination orderings of chordal powers of graphs, Discrete Mathematics 158 (1996) 273-278.

[3] E. Dahlhaus And P. Duchet, On strongly chordal graphs, Ars Combinatoria 24B (1987) 23-30.

[4] R.B. Eggleton, P. ERdôs And D.K. Skilton, Coloring the real line, Journal of Combinatorial Theory B39 (1985) 86-100.

[5] F. HaRARY AND S. HedeTniemi, The achromatic number of a graph, Journal of Combinatorial Theory 8 (1970) 154-161.

[6] F. Hughes And G. MacGillivray, The achromatic number of graphs: A survey and some new results, Bull. Inst. Comb. Appl. 19 (1997) 27-56.

[7] R. W. IRving And D. F. Manlove, The b-chromatic number of a graph, Discrete Applied Mathematics 91 (1999) 127-141.

[8] H.Kheddouci, J.F.Saclé And M.WoźniaK, Packing of two copies of a tree into its fourth power, Discrete Mathematics 213 (1-3) (2000) 169-178.

[9] M.Kouider AND M.MAHEO, Some bounds for the b-chromatic number of a graph, Discrete Mathematics 256, Issues 1-2, (2002) 267-277.

[10] D.E. KnUth, The Art of Computer Programming, Vol. 3 Addison-Wesley, Reading. MA. 1975.

[11] J. Kratochvíl, Z. TuZa And M. Voigt, On the b-chromatic number of graphs, Proceedings WG'02 - 28th International Workshop on Graph-Theoretic Concepts in Computer Science, Cesky Krumlov, Czech Republic, volume 2573 of Lecture Notes in Computer Science. Springer Verlag 2002.

[12] I.Z. Ruzsa, Z. TuZa And M. Voigt, Distance Graphs with Finite Chromatic Number, Journal of Combinatorial Theory B85 (2002) 181-187.

[13] X. ZHU, Pattern periodic coloring of distance graphs, Journal of Combinatorial Theory B73 (1998) 195-206. 